\title{
SIMULATION-BASED HYBRID APPROACH TO ROBUST MULTI-ECHELON INVENTORY POLICIES FOR COMPLEX DISTRIBUTION NETWORKS
}

\author{
Fang, D. J. ${ }^{*} \& \mathrm{Li}, \mathrm{C}$.* \\ *Sino-German College for Graduate Study, Tongji University, Shanghai, China \\ ${ }^{* *}$ Fraunhofer Institute for Material Flow and Logistics, Dortmund, Germany \\ E-Mail: fang.dianjun@cdhk.tongji.edu.cn,fang@iml.fhg.de
}

\begin{abstract}
In today's dynamic market numerous dynamic influencing factors have seriously aggravates the difficulties of inventory planning of the complex distribution networks. This paper proposes a simulation-based hybrid approach for the optimization of the inventory policies in complex distribution networks. The initial multi-echelon inventory policies are handed over to a simulation model, which is capable of modelling complexity and uncertainties of the distribution network and simulating them under respective scenarios. Through comprehensively analysing the KPIs (logistic service level and logistic costs) of this set of multi-echelon inventory policies, their levels of robustness can be clearly ascertained. Based on the simulation results, a metaheuristic-based optimizer regenerates improved (more robust) multi-echelon inventory policies, which are once again comprehensively and precisely evaluated through simulation. This closed feedback loop forms a simulation optimization process that enables the autonomous evolution of multi-echelon inventory policies of complex distribution networks. Since the simulation results can truly reflect the performance of certain inventory policies in real market environment, the new Simulation-Based Hybrid Approach is quite useful for decision making process.

(Received, processed and accepted by the Chinese Representative Office.)
\end{abstract}

Key Words: Simulation, Metaheuristic, Evolutionary Algorithms, Inventory Policies, Distribution Network

\section{INTRODUCTION}

In today's dynamic market, distribution networks are always finding themselves in a continuously changing environment. Numerous driving forces of changes come not only from outside such as volatile customer demand, shortened product life cycle and individualized customer wishes [1]; but also from inside, like short-of-stock, delay or suspension [2]. These mutually overlapping and interacting factors cause turbulent changes in the environment that distribution networks are facing and impose strong pressures for adaptation on every level of distribution networks [3]. Consequently, today's distribution networks are highly expected to be adaptive or robust, which means that they will not leave the specified (optimal) logistic cost and logistic service level corridor even under the changing conditions [4].

Inventory is one important type of configuration parameter of distribution networks. On the one hand, as "an aggregate resource that spans and connects all nodes" in distribution networks, inventory serves as an important means to "separate or buffer one location in the distribution channel from another and the discontinuity of customer demand from available inventories", so that the customers' wishes of time, location, quantity, quality and price can be fulfilled [5]. On the other hand, large amounts of resources including capital, labour and space, are tied up in holding the inventories [5-8], which takes up a significant proportions of the logistics costs [2]. It is imperative to carefully conduct the inventory planning so that optimal inventory policies can be made out. However due to the turbulent changes in today's 
dynamic market, the behaviours and status of the distribution network are completely unforeseeable and incomprehensible [9], which has largely aggravated the mismatch between supply and demand. Correspondingly, the optimized inventory policies are strongly expected to be able to withstand these dynamic influences. The inventory policies should be robust enough to cope with various uncertainties and dynamics.

The multi-echelon inventory model used in this study comes from Klingebiel and Li [10], which considers a general multi-echelon distribution network comprising several warehouses (square shaped symbols) in each echelon and multiple stock points (triangle shaped symbols) in each warehouse (see Fig. 1). Every warehouse is regarded as storing the same variety of products and each stock point corresponds to exactly one kind of product. The echelon that faces directly end customer is regarded as the $1^{\text {st }}$ echelon (dotted line box). Every stock point is assumed to be continuously reviewed and implement an $(R, Q)$ inventory policy, which means that when the inventory position, expressed as the physical inventory plus the stock on order minus backorders, is equal to or drops below its reorder point $R$, a replenishment order is placed towards its supplier with the order quantity $Q[7,8]$.

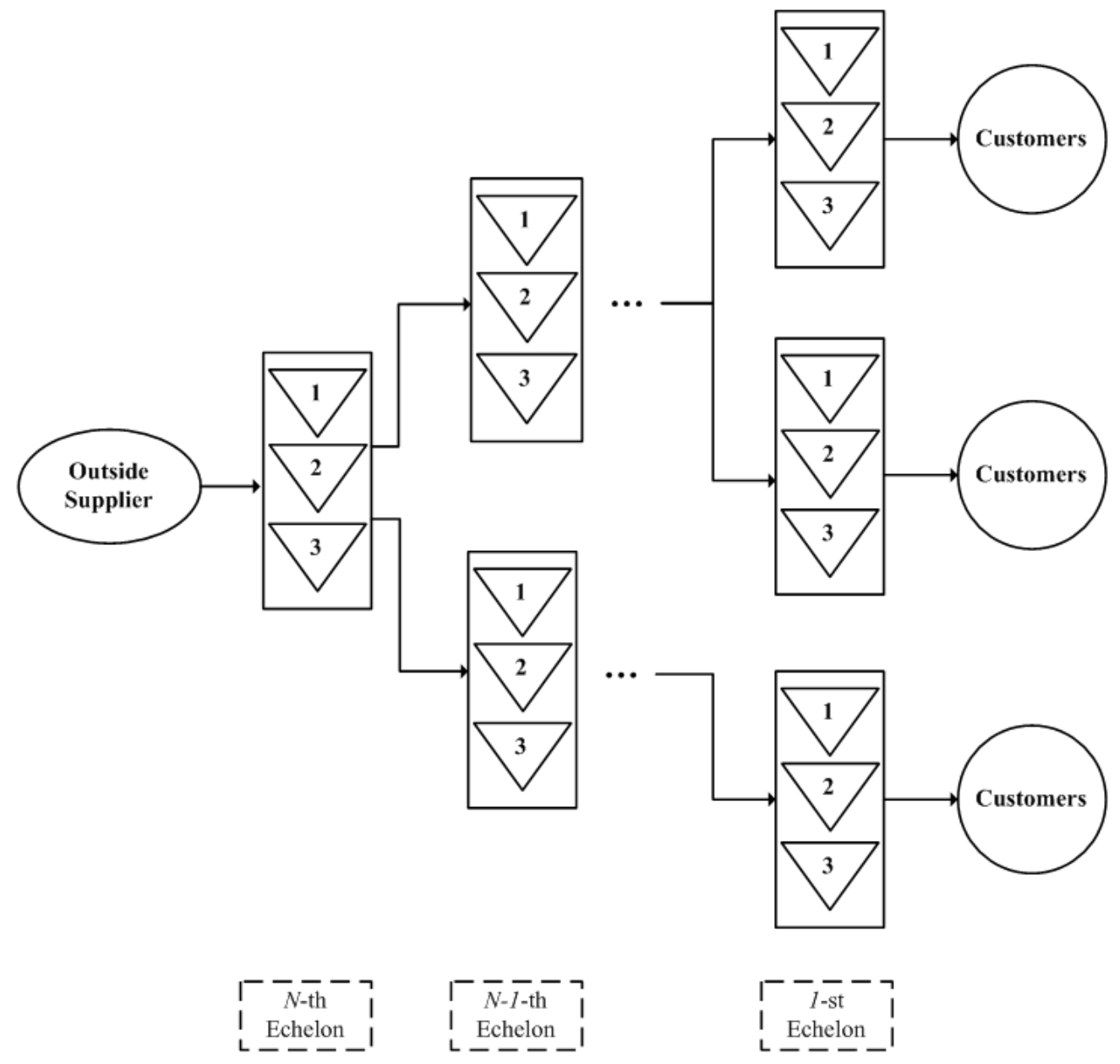

Figure 1: A general multi-echelon distribution network.

\section{STATE-OF-THE-ART APPROACHES FOR OPTIMIZATION OF INVENTORY POLICIES}

Traditional approaches for optimized inventory policies are mostly based on simplified deterministic single- or multi-echelon inventory models, which have provided a deep insight 
into the mechanism of inventory controls throughout the distribution network $[7,11]$. But they are no longer capable of delivering the desired results or even greatly deteriorate the performance of the complex distribution networks in today's dynamic environment, leading to either low logistic service level or high logistic costs [10].

Besides the traditional analytical approaches, another type of approaches for analysing complex distribution networks is simulation, which is increasingly utilized with the rapid development of information technology [9, 12]. Apart from the traditional view that simulation serves only as "a descriptive tool in the modelling and analysis of a wide variety of complex real systems", recent advancement in optimization approach and computing technology make it "a prescriptive tool in support decision making" [13]. In Barton's work, the simulation-based optimization is defined as "repeated analysis of the simulation model with different values of design parameters, in an attempt to identify best simulated system performance" [14]. In other words, the optimal (or sub-optimal) design parameters of the real system are "determined by the simulation optimization process, rather than in an ad hoc manner based on qualitative insights gained from exercising the simulation model" [14]. It seems pretty simple to understand, however, a wide variety of difficulties have arisen when applying a simulation-based optimization process in practice. First of all, there typically exist a large numbers of different types of parameters that are subject to optimization. For this kind of problems, the optimization itself is not an easy task. Thus, the effort of accelerating convergence speed or finding an improving direction often ends in vain.

All the mentioned analytical, simulation and simulation-based optimization approaches on inventory policies for distribution networks have failed to solve the difficulties resulting from the inherent complexity, volatile customer demand and multi-objective optimization of the complex distribution networks. To overcome these challenges, a new simulation- based hybrid approach is introduced in this paper.

\section{INTRODUCTION OF THE NEW SIMULATION HYBRID APPROACH}

Based on the above analysis, a new hybrid approach is developed which is composed of two closely interrelated components: a simulation-based evaluation of the multi-echelon inventory policies under the context of dynamics and uncertainty, and a metaheuristic-based optimizer that improves these multi-echelon inventory policies on the basis of simulation results. The schematic diagram of this hybrid approach is illustrated in Fig. 2.

The hybrid approach starts with generation of an initial generation of multi-echelon inventory policies, which will be handed over to a simulation model. The professional simulation tool OTD-NET [15], developed by Fraunhofer-Institut für Materialfluss und Logistik (IML), is applied, which is capable of modelling complexity and uncertainties of the distribution network and simulating them under respective scenarios (i.e. market dynamics). Through comprehensively analysing the KPIs (in the category of logistic service and logistic costs) of this set of multi-echelon inventory policies, their levels of robustness can be clearly ascertained.

Based on these results, a metaheuristic-based optimizer regenerates improved (more robust) multi-echelon inventory policies, which are once again comprehensively evaluated through simulation. This closed feedback loop forms a simulation optimization process that enables the autonomous evolution of multi-echelon inventory policies. The iteration continues until given termination criteria are finally satisfied. Since each multi-echelon inventory policy has been carefully tested under real dynamics, the robustness level of the resulting multiechelon inventory policies can be determined. In other words, under these multi-echelon inventory policies, the distribution network can maintain a specified optimality (or suboptimality) in spite of dynamic influencing factors. 


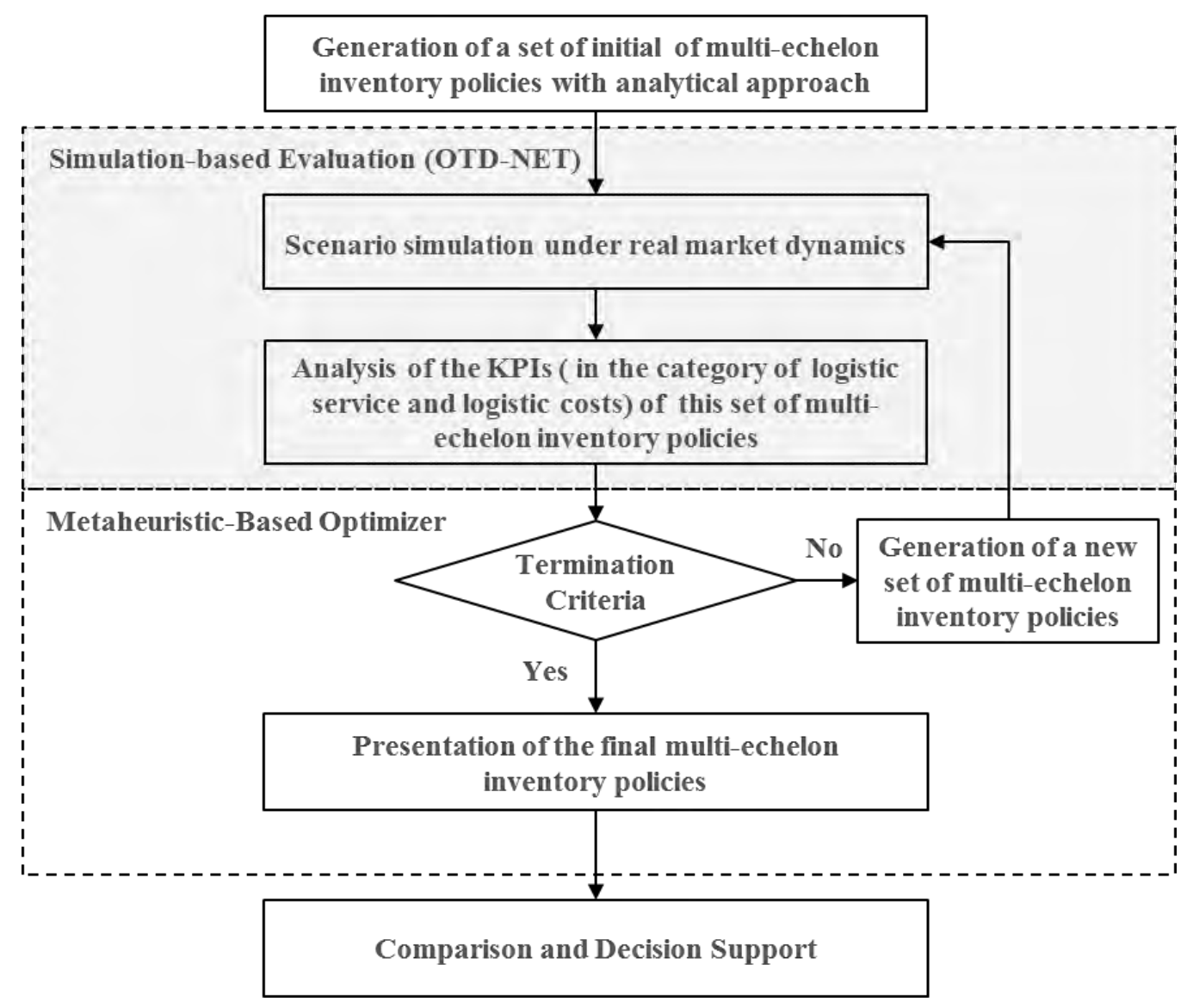

Figure 2: A new hybrid approach to robust multi-echelon inventory policies for distribution networks.

\section{SIMULATION-BASED EVALUATION OF MULTI-ECHELON INVENTORY POLICIES}

In this study, the excellent simulation tool OTD-NET is applied. With a holistic view of modelling and simulating of complex production and logistics networks, this simulation tool can deliver in-depth insights into information and material flow, stock levels, stability of the network, boundary conditions and restrictions [16]. Since introduction, its effectiveness has been demonstrated in many logistic projects (e.g. ILIPT, VW Nutzfahrzeug), especially in the simulation field where information flow is tightly coupled with material flow [15].

Having implemented a novel and object-oriented modelling methodology, OTD-NET allows the user to map all relevant network elements (e.g. distribution channels, buffers, customers, and dealers) as well as many influencing parameters (e.g. inventory policies, transportation plan and time windows) in a selectable level of details. As a discrete-event simulation tool, OTD-NET offers adequate ability to process and record simulation data, which is essential to conduct effective statistical analysis according to simulation results [15]. To facilitate data exchange, OTD-NET provides not only a generic XML-interface, which enables to import data from other independent components; but also auxiliary executable programs, which assist the automatic control of simulation from other independent program modules.

To completely represent the inventory flow throughout the distribution network from outside supplier (or plant) to end customers, the simulation model of $(R, Q)$ inventory policies for the distribution network is conceptualized mainly from the following three aspects. 
A. Conception of physical structure

In a complex distribution network, products have to go through multiple echelons before arriving at end customers. In each echelon there exist multiple warehouses, each of which is again composed of several stock points. As an object-oriented simulation tool, OTD-NET has already incorporated "plant" and "customer" classes, so that these objects can be directly instantiated in the simulation model. Classes like "warehouse" or "distributor centre", however, have not offered in its entire class structure of OTD-NET. Instead, another innovative concept - "distribution channel" is introduced, which implies that a product is always in a flowing process until it has reached its ultimate customers. A "distribution channel" can either represent an actual route channel with starting point and end point located in different positions, or stands for an abstract channel with those merged together, i.e. a concrete logistic node (e.g. a warehouse) [16]. Not intuitive, though, this kind of model description has the advantage of flexibility, which could model any kinds of physical structures of distribution networks with as few types of classes as possible.

To give a more vivid impression, the OTD-NET model for the exemplary distribution network used in case study is illustrated in Fig. 3. Besides the single plant at the beginning and multiple customers at the end of the distribution network, all the transportation routes and logistic nodes (i.e. distribution centres; EDC and RDCs in Fig. 3) are modelled by distribution channels (chevron shaped symbol in Fig. 3), of which the logistic nodes are distinguished in orange colour. With these limited building stocks, OTD-NET can model a distribution network with arbitrary variations of vertical and horizontal structures.

B. Conception of $(R, Q)$ inventory policies

According to the definition of $(R, Q)$ inventory policies, a replenishment order with size $Q$ will not be placed towards its supplier until the inventory position, expressed as the physical inventory plus the stock on order minus backorders, of one stock point is equal to or drops below its reorder point $\mathrm{R}$ [7].

As discussed in subsection A, there are no concrete objects in OTD-NET for "storing" and "recording" inventories. So such $(R, Q)$ inventory policies cannot be directly modelled as by some classical simulation tools. To solve this problem, OTE-NET has introduced a special object "transportation ticket (TT)", which is utilized to regulate the transportation of items (e.g. finished goods, work-in-process, and materials) through "distribution channel". With the "transportation ticket", a "distribution channel" can determine if an item is allowed to pass through.

The batch-sized order $Q$ are realized through the accumulation of "transportation ticket (TT)", which means that a stock point will not send TTs to its predecessor until its order size has reached a predefined amount (i.e. order quantity $Q$ ). When the required items are available in its predecessor, they will be delivered to their destinations in the rule of first-in, first out (FIFO). The reorder point $R$ is realized through initializing the amount of inventories in each stock point to be the sum of its reorder point and order quantity, i.e. $R+Q$. When an item has left this stock point, it is transformed into a TT by the "Type Converter" object (the square shaped symbol in Fig. 3). If these accumulated TTs have reached a threshold value (i.e. batch-size), they will be sent together to its predecessor, i.e. in a batch-sized order (see the upper part of Fig. 3). If there are multiple successors for a stock point, the "Routing Table" objects (the diamond shaped symbol in Fig. 3) will lead the delivery according to the item's TT which has its destination information.

As a result, each stock point in the distribution network will send its batch-sized order only when its inventory position has dropped to its reorder point. That is to say, the multiechelon $(R, Q)$ inventory policies have been successfully modelled with OTD-NET. 
C. Conception of Volatile Customer Demand

Since this simulation model is desired to fully reproduce the volatile customer demand, the detailed demand forecast scenarios in daily granularity are applied. With the characteristics of both stochastic and time-varying, these demand scenarios can demonstrate strong seasonality, interval interruption and abrupt changes.

Yet, a practical problem arises when modelling these demand scenarios with OTD-NET because this simulation tool is originally developed for simulating order processing process in automobile industry [16], which has already assigned an item its customer's information (e.g. Build-to-Order). In a distribution network, nonetheless, an item does not surely know its "end customer" in advance, which makes the standard modelling method of OTD-NET not eligible.

Here, the modelling tricks are applied to realize the daily demand scenarios in OTD-NET. Firstly, a set of virtual customers, which do not generate orders, are developed as the sink of material flow processes in the distribution network. The real customer demand data is injected from a XML file through auxiliary channels (also are "distribution channel" objects, see the lower part of Fig. 3). Then, the detailed orders are created from random mechanisms of OTDNET according to the given demand amount in each simulation day. Consequently, the corresponding scenarios of daily varying demand are completely reproduced with OTD-NET, which is sufficient to reflect the market dynamics.

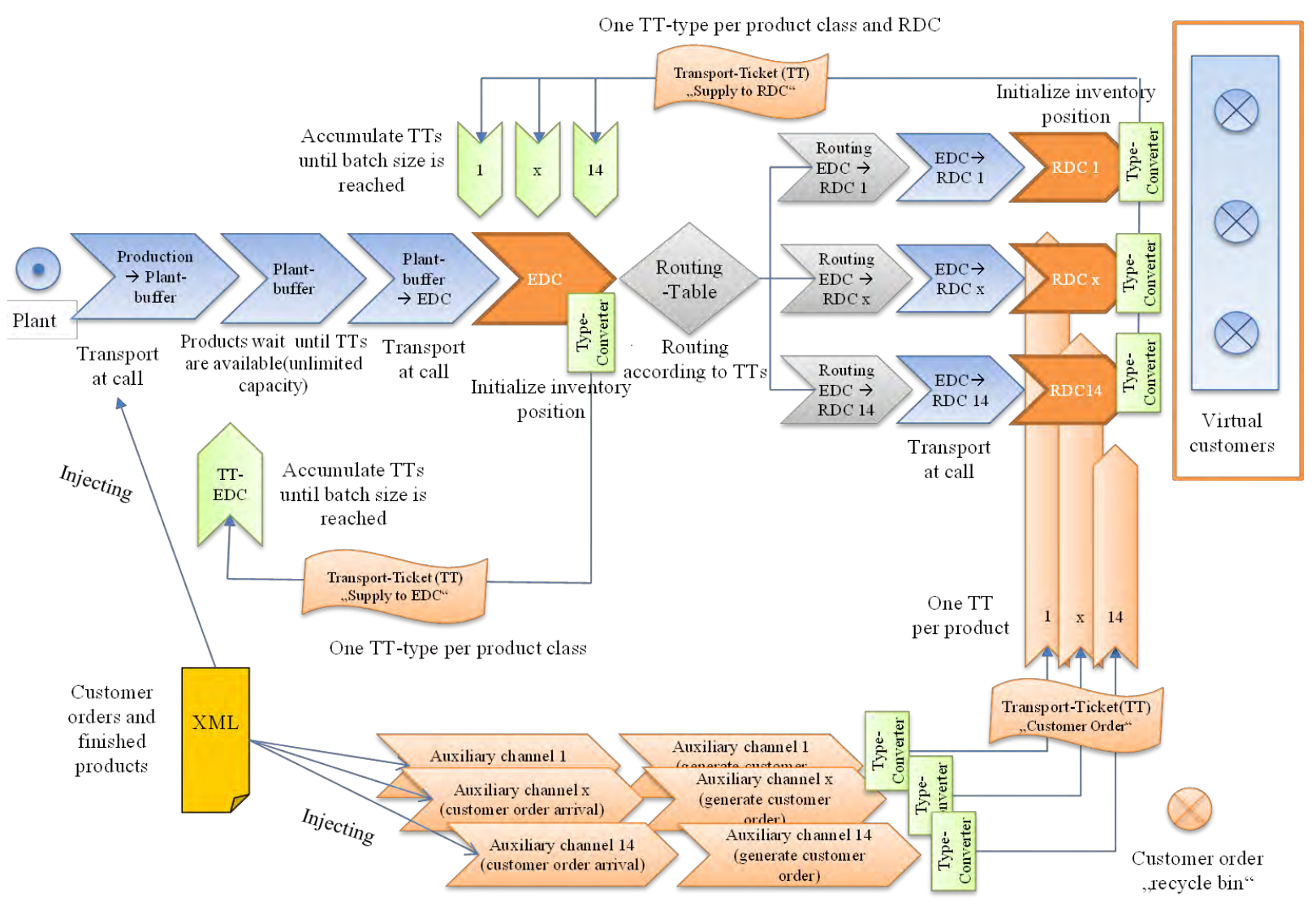

Figure 3: OTD-NET modelling structure of an exemplary distribution network.

\section{METAHEURISTIC-BASED OPTIMIZER OF MULTI-ECHELON INVENTORY POLICIES FOR DISTRIBUTION NETWORKS}

The metaheuristic-based optimizer is designed on the basis of a special evolutionary algorithm NSGA-II, which is one of the most widely used multi-objective evolutionary algorithms $[17,18]$. 
With regard to the optimization of multi-echelon inventory policies, there exist two types of parameters in this metaheuristic-based optimizer. One is the running parameters, which are used during the optimization process; the other is the termination parameters, which are employed to stop the optimization process. In an evolutionary algorithm, running parameters usually include population size, crossover rate and mutation rate, while the frequently used termination parameters are target fitness value and maximum generation. However, not all of these parameters have to appear in a single algorithm, which are determined in accordance with the distinct requirements of a problem. Conventional evolutionary algorithms usually transform a multi-objective optimization problem into a single-objective one and then evaluate the fitness of individuals according to this objective function. In contrast, NSGA-II tries to simultaneously assess individuals in all dimensions without any artificial alteration or distortion, which defines two criteria for evaluating individual: quality (absolute fitness) and distribution (relative fitness) [18].

In this multi-echelon inventory policy problem, there are two incommensurable and conflicting objectives. Inventory costs are measured in monetary unit and are the lower the better, whereas fill rate is evaluated as percentage and is the higher the better. Since it is impossible to compare two multi-echelon inventory policies directly, the concepts of Pareto rank and crowding distance are employed to assess different multi-echelon inventory policies.

After the mating pool has been formed, a new set of multi-echelon inventory policies (i.e. population) can be generated. Each time two individuals are selected randomly without replacement from the mating pool to perform crossover and mutation. There are numerous kinds of approaches to conduct crossover and mutation. In this research the simulated binary crossover (SBX) and polynomial mutation [19] are applied according to the standard NSGAII. In this customized evolutionary algorithm, the procedures of polynomial mutation have been embedded into SBX crossover to reduce the probability of mutation and thus stabilize the optimization process.

SBX crossover is aimed to simulate the sharing centroid property, which originally exists in the case of binary code crossover, after a real code crossover [20]. An important variable called spread factor $\beta_{i}$ is used to determine the amount of the change of the difference between two $i^{\text {th }}$ genes that is caused by crossover, i.e. [20].

$$
\beta_{i}=\left|\frac{c_{i}^{1}-c_{i}^{2}}{p_{i}^{1}-p_{i}^{2}}\right|
$$

where $c_{i}^{1}$ and $c_{i}^{2}$ are $i^{\text {th }}$ genes of the two children while $p_{i}^{1}$ and $p_{i}^{2}$ are those of the two parents respectively. If $\beta_{i}<1$, the operator is called a contracting crossover; if $\beta_{i}>1$, it is called an expanding crossover; and if $\beta_{i}=1$, it is called a stationary crossover [18]. Since the stationary crossover is expected to occur frequently whereas the contracting or expanding crossover should appear only with a relatively low probability, they proposed a probability density function for the random variable $\beta_{i}$ as below [18]:

$$
P\left(\beta_{i}\right)= \begin{cases}0.5(n+1) \beta_{i}{ }^{n} & \beta_{i} \leq 1 \\ 0.5(n+1) \frac{1}{\beta_{i}{ }^{n+2}} & \beta_{i}>1\end{cases}
$$

where $n$ is a control parameter. In this probability distribution, the larger $n$ is, the higher probability $\beta_{i}$ has of being close to 1 [18].

To generate the random number $\beta_{i}$ that follows the above probability density function, the most frequently used method is the inverse transformation that generates it from the uniformly distributed random number $u_{i} \sim \mathrm{U}(0,1)$, i.e. [18]:

$$
\beta_{i}= \begin{cases}\left(2 u_{i}\right)^{\frac{1}{n+1}} & u_{i} \leq 0.5 \\ {\left[2\left(1-u_{i}\right)\right]^{-\frac{1}{n+1}}} & u_{i}>0.5\end{cases}
$$


Correspondingly, the $i^{\text {th }}$ gene of these two children is given by [18]:

$$
\begin{aligned}
& c_{i}^{1}=0.5\left(p_{i}^{1}+p_{i}^{2}\right)+0.5 \beta_{i}\left(p_{i}^{1}-p_{i}^{2}\right) \\
& c_{i}^{2}=0.5\left(p_{i}^{1}+p_{i}^{2}\right)+0.5 \beta_{i}\left(p_{i}^{2}-p_{i}^{1}\right)
\end{aligned}
$$

The polynomial mutation is based on the polynomial distribution, of which the mean equals to the current value and the variance is a function of the distribution control ter $n$ [21]. To carry out mutation, a perturbance factor $\delta_{i}$ is defined to measure the amount of change in the $i^{\text {th }}$ gene, i.e. [18]:

$$
\delta_{i}=\frac{c_{i}-p_{i}}{\Delta_{\max }}
$$

where $c_{i}$ and $p_{i}$ are the $i^{\text {th }}$ gene of the child and parent, and $\Delta_{\max }$ is the largest permissible perturbance of the $i^{\text {th }}$ gene.

The perturbance factor $\delta_{i}$ follows a polynomial distribution, which is given by [21]:

$$
P\left(\delta_{i}\right)=0.5(n+1)\left(1-\left|\delta_{i}\right|\right)^{n}
$$

where $n$ is a control parameter and $\delta_{i} \in[-1,1]$. Moreover, the larger $n$ is, the higher probability $\delta_{i}$ has of being close to 0 [18]. The random variable $\delta_{i}$ can be generated with the similar inverse transformation as the spread factor $\beta_{i}$ in SBX crossover, i.e. [18]:

$$
\delta_{i}= \begin{cases}\left(2 u_{i}\right)^{\frac{1}{n+1}}-1 & u_{i} \leq 0.5 \\ 1-\left[2\left(1-u_{i}\right)\right]^{\frac{1}{n+1}} & u_{i}>0.5\end{cases}
$$

Then, the mutated value of the $i^{\text {th }}$ gene is given as below [18]:

$$
c_{i}=p_{i}+\delta_{i} * \Delta_{\max }
$$

Besides, the positive and negative perturbance factors are defined:

- positive perturbance factor:

- negative perturbance factor:

$$
\left[\delta_{i}\right]^{+}= \begin{cases}1-\left(2 u_{i}\right)^{\frac{1}{n+1}} & u_{i} \leq 0.5 \\ 1-\left[2\left(1-u_{i}\right)\right]^{\frac{1}{n+1}} & u_{i}>0.5\end{cases}
$$

$$
\left[\delta_{i}\right]^{-}= \begin{cases}\left(2 u_{i}\right)^{\frac{1}{n+1}}-1 & u_{i} \leq 0.5 \\ {\left[2\left(1-u_{i}\right)\right]^{\frac{1}{n+1}}-1} & u_{i}>0.5\end{cases}
$$

The metaheuristic-based optimizer is developed to work in together with the simulation module, so that a simulation optimization process can be carried out. Since the metaheuristicbased optimizer is designed on the basis of a special evolutionary algorithm NSGA-II, the main task of the implementation is to enable the autonomous evolution of multi-echelon inventory policies according to their robustness levels that are evaluated through simulation.

\section{CASE STUDY - PROOF OF CONCEPT}

In the exemplary distribution network, finished products are produced in one German plant and sold in selected European markets. To deliver the products to end customers, a European distribution centre (EDC) and 14 regional distribution centres (RDCs) have been constructed. Respective forecast scenarios are used to reflect the volatility of the markets (see Fig. 4 for example of Article I).

The daily on-hand inventory level of the selected single distribution centres (e.g. EDC, RDC04 \& RDC06) are illustrated in Fig. 5. When the line touches the X-axis, stock-out situations occur. The service level (fill rate) of the EDC in average $88.2 \%$ may be acceptable, if its influences on downstream distribution centres are not taken into account. Yet, the EDC has suffered from stock out for nearly two months (54 days). Such serious stock-out is not 
allowed to occur in practice, which might result in a huge amount of manual interventions or a great loss of customers. Thus, simulation can present more precise and comprehensive evaluation of different multi-echelon inventory policies, which is quite beneficial to decision making.

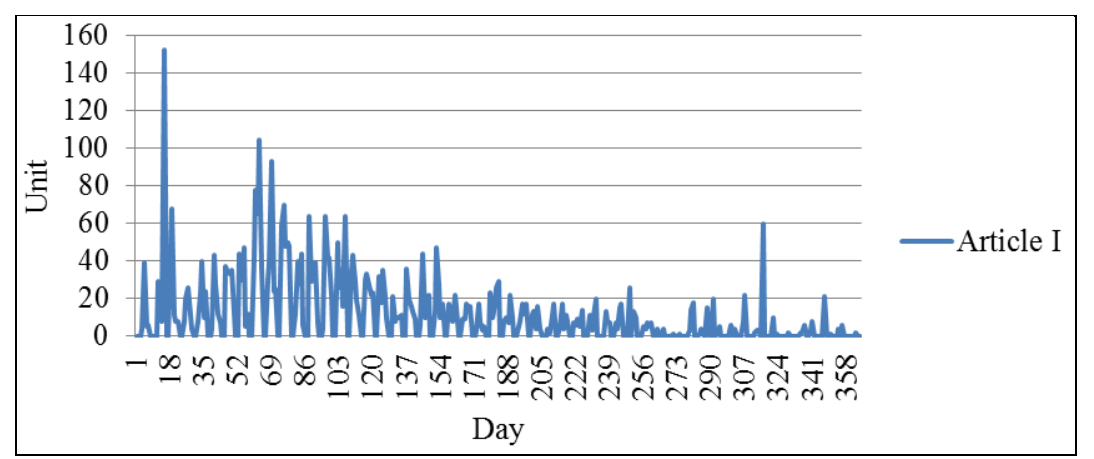

Figure 4: Daily demand of Article I aggregated over all 14 markets.

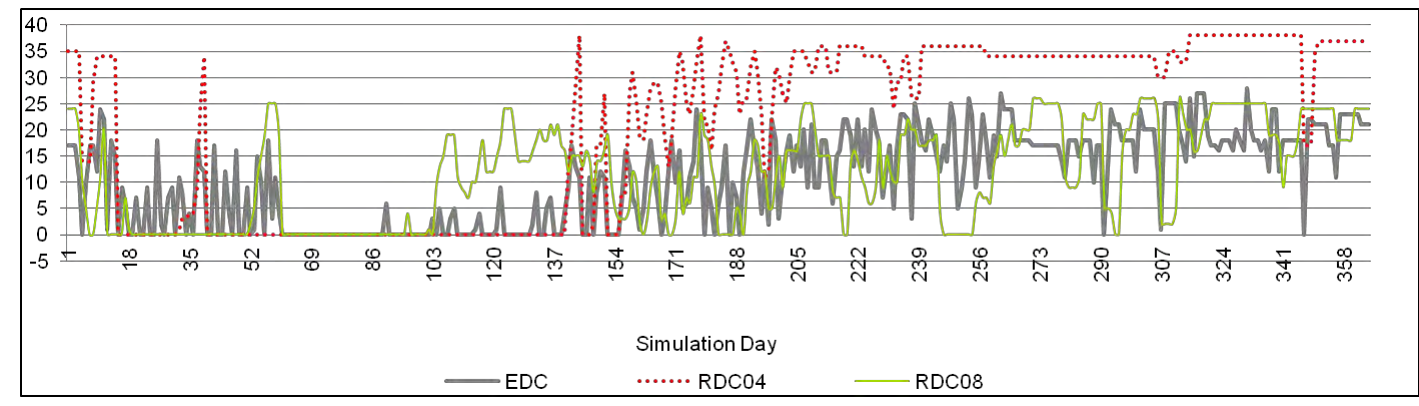

Figure 5: Monthly demand of Article I aggregated over all 14 markets.

The new simulation-based hybrid approach is applied in the exemplary distribution network. The evolutionary process of multi-echelon inventory policies is demonstrated in Fig. 6 . Here, the accepted corridor of inventory costs is specified between 500 and 700 monetary units, that is to say, the optimized multi-echelon inventory policies should only be accepted when the resulting inventory costs of the distribution network have not left this corridor. It can be seen that the multi-echelon inventory policies after $50^{\text {th }}$ generations have brought about remarkably better performance than those after $10^{\text {th }}$ generations, which implies that the logistic service level (i.e. fill rate) and the logistic costs (i.e. inventory costs) of the distribution network are simultaneously enhanced. The improvement obtained from the $50^{\text {th }}$ generation to $90^{\text {th }}$ generation (triangle shaped symbol in Fig. 6), however, is not that distinguish. This can be explained by the fact that as the multi-echelon inventory policies become increasingly robust, little room is left for improvement. From another perspective, the simulation results have demonstrated that the metaheuristic-based optimizer can find out highly qualified multi-echelon inventory policies in relatively short computational time, i.e. it is quite efficient. The analytical model is applied to generate an initial generation of multiechelon inventory policies. The quality of the initial generation of multi-echelon inventory policies is nevertheless irrelevant for the simulation-based hybrid approach. All the finally optimized multi-echelon inventory policies (circle shaped symbol) have formed a Pareto optimal front, which means that for almost each level of logistic costs (i.e. inventory costs) there exists a multi-echelon inventory policy that can bring about the best logistic service level (i.e. fill rate). Decision makers can select robust multi-echelon inventory policies and implement them in practice according to their specified level of logistic performance and logistic costs. 


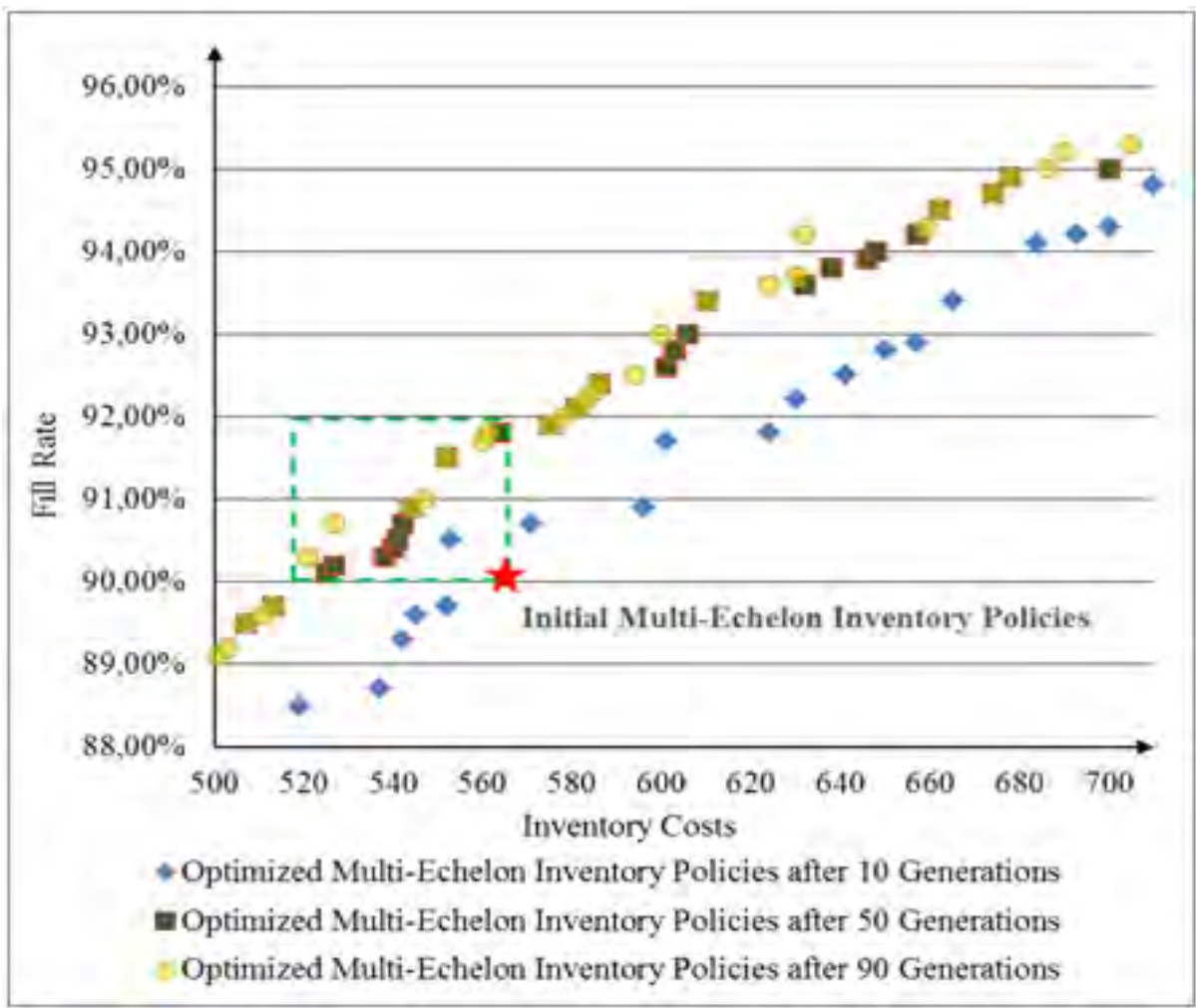

Figure 6: Simulation results of the initially and finally optimized multi-echelon Inventory Policies.

\section{CONCLUSIONS}

Due to the inherent complexity and external dynamics of a distribution network in today's dynamic market, the traditional approaches have failed to propose robust multi-echelon inventory policies that can lead to high logistic service with low logistic costs under market dynamics. In this paper a new hybrid approach for deriving robust multi-echelon inventory policies for distribution networks has been developed, which integrates a simulation-based evaluation of multi-echelon inventory policies with a metaheuristic-based optimizer together. To evaluate the robustness level of these inventory policies under market dynamics, the initial multi-echelon inventory policies are handed over to an OTD-NET simulation model. Based on the simulation results, i.e. the robustness level of the proposed multi-echelon inventory policies, a metaheuristic-based optimizer is developed to regenerate improved (more robust) multi-echelon inventory policies, which are once again automatically evaluated through simulation. Instead of a single optimal multi-echelon inventory policy, multiple Pareto optimal multi-echelon inventory polices are proposed, which is quite beneficial to the practical decision making. This hybrid approach has been applied to an industrial case, in which a set of robust multi-echelon inventory polices are proposed. These optimized multiechelon inventory policies maintain a specified optimality (or sub-optimality) despite anticipated market dynamics and are quite superior to existing approaches.

\section{ACKNOWLEDGEMENT}

The research has been supported by German DFG (project: MMeAS - KU 619/18-1) and China Scholarship Council. 


\section{REFERENCES}

[1] Nyhuis, P.; Reinhart, G.; Abele, E. (eds.) (2008). Wandlungsfähige Produktionssysteme: Heute die Industrie von morgen gestalten, PZH Produktionstechnisches Zentrum, Garbsen, 20-28

[2] Rushton, A.; Oxley, J.; Croucher, P. (2001). The handbook of logistics and distribution management, $2^{\text {nd }}$ ed., reprinted, Kogan Page, London, 3-10, 35-48, 73-82, 181-197, 200-213

[3] Cisek, R.; Habicht, C.; Neise, P. (2002). Gestaltung wandlungsfähiger Produktionssysteme, Zeitschrift für wirtschaftlichen Fabrikbetrieb, Vol. 97, No. 9, 441-445

[4] Kuhn, A.; Klingebiel, K.; Schmidt, A.; Luft, N. (2011). Modellgestütztes Planen und kollaboratives Experimentieren für robuste Distributionssysteme, 24. HAB-Forschungsseminar der Hochschulgruppe für Arbeits- und Betriebsorganisation, 177-198

[5] Ross, D. F. (1996). Distribution: Planning and control, Chapman \& Hall Materials Management/Logistics series, New York, Chapman \& Hall, 4-23, 213-237, 388-412

[6] Mangan J.; Lalwani, C.; Butcher, T. (2010). Global logistics and supply chain management, Wiley, Chichester

[7] Axsäter, S. (2006). Inventory control, $2^{\text {nd }}$ ed., International Series in Operations Research \& Management Science, Vol. 90, Springer, New York

[8] Zipkin, P. H. (2000). Foundations of inventory management, McGraw-Hill, Boston

[9] Kuhn, A., Hellingrath, B. (2002). Supply Chain Management: Optimierte Zusammenarbeit in der Wertschöpfungskette, Springer, Berlin, 1-35, 87-124, 213-228, doi:10.1007/978-3-662-10141-4

[10] Klingebiel, K.; Li, C. (2011). Optimized multi-echelon inventory policies in robust distribution networks, Proceedings of $25^{\text {th }}$ European Conference on Modelling and Simulation, 573-579

[11] Tempelmeier, H. (2006). Inventory management in supply networks: Problems, models, solutions, Books on Demand, Norderstedt

[12] Chopra, S.; Meindl, P. (2010). Supply chain management: Strategy, planning, and operation, $4^{\text {th }}$ ed., Prentice Hall, Boston, 85-121, 263-344

[13] Tekin, E., Sabuncuoglu, I. (2004). Simulation optimization: A comprehensive review on theory and applications, IIE Transactions, Vol. 36, No. 11, 1067-1081, doi:10.1080/ 07408170490500654

[14] Barton, R. R.; Meckesheimer, M. (2006). Metamodel-based simulation optimization, Henderson S. G.; Nelson, B. L. (eds.), Handbooks in Operations Research and Management Science, Vol. 13, Elsevier, Amsterdam, 535-574

[15] Klingebiel, K. (2009). Entwurf eines Referenzmodells für Built-to-order-Konzepte in Logistiknetzwerken der Automobilindustrie, Dissertation, Verlag Praxiswissen, Dortmund

[16] Wagenitz, A. (2007). Modellierungsmethode zur Auftragsabwicklung in der Automobilindustrie, Dissertation, Verlag Praxiswissen, Dortmund

[17] Konak, A.; Coit, D. W.; Smith, A. E. (2006). Multi-objective optimization using genetic algorithms: A tutorial, Reliability Engineering \& System Safety, Vol. 91, No. 9, 992-1007, doi:10.1016/j.ress.2005.11.018

[18] Yu, X.; Gen, M. (2010). Introduction to evolutionary algorithms, Decision Engineering Series, Springer, London, doi:10.1007/978-1-84996-129-5

[19] Deb, K.; Pratap, A.; Agarwal, S.; Meyarivan, T. (2002). A fast and elitist multiobjective genetic algorithm: NSGA-II, IEEE Transactions on Evolutionary Computation, Vol. 6, No. 2, 182-197, doi:10.1109/4235.996017

[20] Deb, K.; Agrawal, R. B. (1995). Simulated binary crossover for continuous search space, Complex Systems, Vol. 9, No. 2, 115-148

[21] Deb, K.; Goyal, M. (1996). A combined genetic adaptive search (GeneAS) for engineering design, Computer Science and Informatics, Vol. 26, No. 4, 30-45 